\title{
Airborne Subscale Transport Aircraft Research Testbed - Aircraft Model Development
}

\author{
Thomas L. Jordan ${ }^{*}$ William M. Langford ${ }^{\dagger}$ and Jeffrey S. Hill ${ }^{\ddagger}$ \\ NASA Langley Research Center, Hampton, VA 23681
}

\begin{abstract}
The Airborne Subscale Transport Aircraft Research (AirSTAR) testbed being developed at NASA Langley Research Center is an experimental flight test capability for research experiments pertaining to dynamics modeling and control beyond the normal flight envelope. An integral part of that testbed is a $5.5 \%$ dynamically scaled, generic transport aircraft. This remotely piloted vehicle (RPV) is powered by twin turbine engines and includes a collection of sensors, actuators, navigation, and telemetry systems. The downlink for the plane includes over 70 data channels, plus video, at rates up to $250 \mathrm{~Hz}$. Uplink commands for aircraft control include over 30 data channels. The dynamic scaling requirement, which includes dimensional, weight, inertial, actuator, and data rate scaling, presents distinctive challenges in both the mechanical and electrical design of the aircraft. Discussion of these requirements and their implications on the development of the aircraft along with risk mitigation strategies and training exercises are included here. Also described are the first training (non-research) flights of the airframe. Additional papers address the development of a mobile operations station and an emulation and integration laboratory.
\end{abstract}

\section{Nomenclature}

$\begin{array}{ll}A & =\text { bifilar width } \\ \bar{C} & =\text { mean aerodynamic chord length } \\ C_{h} & =\text { hinge moment coefficient } \\ d_{F S} & =\text { density of air for full scale vehicle } \\ d_{M} & =\text { density of air for model } \\ F S_{l} & =\text { length of full scale vehicle } \\ F S_{w} & =\text { weight of full scale vehicle } \\ g & =\text { gravitational constant } \\ H M & =\text { hinge moment } \\ I & =\text { inertia } \\ K & =\text { scale factor } \\ L & =\text { bifilar length } \\ M_{l} & =\text { length of model } \\ M_{w} & =\text { weight of model } \\ q & =\text { dynamic pressure } \\ S & =\text { control surface area } \\ t & =\text { period } \\ W & =\text { weight }\end{array}$

\section{Introduction}

$\mathrm{T}_{\mathrm{s}=\mathrm{sin}}$ he NASA Aviation Safety and Security Program (AvSSP) was established to develop technologies for improved safety and security of commercial transport aircraft in response to recommendations by the White House Commission on Aviation Safety and Security ${ }^{1}$. The Single Aircraft Accident Prevention (SAAP) Project of the

\footnotetext{
* Systems Engineer, Aeronautic Systems Engineering Branch, M/S 238, thomas.l.jordan@nasa.gov, AIAA Member.

${ }^{\dagger}$ Design Engineer, Aeronautic Systems Engineering Branch, M/S 238, william.m.langford@nasa.gov.

‡ Aerospace Engineer, Aeronautic Systems Engineering Branch, M/S 238, jeffrey.s.hill@nasa.gov.
} 
AvSSP focuses on the development of technologies to reduce aircraft accidents resulting from loss of vehicle control (or upset) as well as failures. According to the National Transportation Safety Board's accident database, $40 \%$ of all commercial aviation fatalities from 1990 - 1996 were due to loss of control. Control Upset Prevention \& Recovery (CUPR) technologies being developed under SAAP provide control under adverse flight conditions in order to accommodate failures, prevent loss of control, and recover control during loss-of-control events. Technologies being developed include enhanced models of vehicle dynamics to characterize upset conditions, failure detection and identification (FDI) algorithms, and adaptive guidance and control (G\&C) laws. The upset dynamics models have been developed for integration into an enhanced aircraft simulation that is being created for improved upset recovery training, and to support the development and evaluation of the FDI and G\&C algorithms ${ }^{2}$. These algorithms are being developed for use onboard transport aircraft for improved situational awareness and control under adverse and upset conditions related to loss-of-control events. Validation of these technologies is therefore critical. Figure 1 shows how an actual loss of control accident goes beyond the normal flight envelope into regions where aerodynamic data is not available from conventional sources.

The AirSTAR testbed has been developed to provide an in-flight capability to validate various CUPR technologies ${ }^{3}$. To accomplish this, researchers at LaRC have undertaken the task of designing, fabricating, and operating a turbine powered, dynamically scaled generic transport model (GTM). While the challenge to design and fabricate this research vehicle was significant, a more encompassing task of building and training an infrastructure to operate the aircraft in a continuing safe and efficient manner also began to evolve. This task included the education and training of a core group of pilots, the development of safety and operational procedures and checklists, the training of essential ground support personnel, and the identification and coordination of test sites external to NASA Langley.

The remainder of this paper is

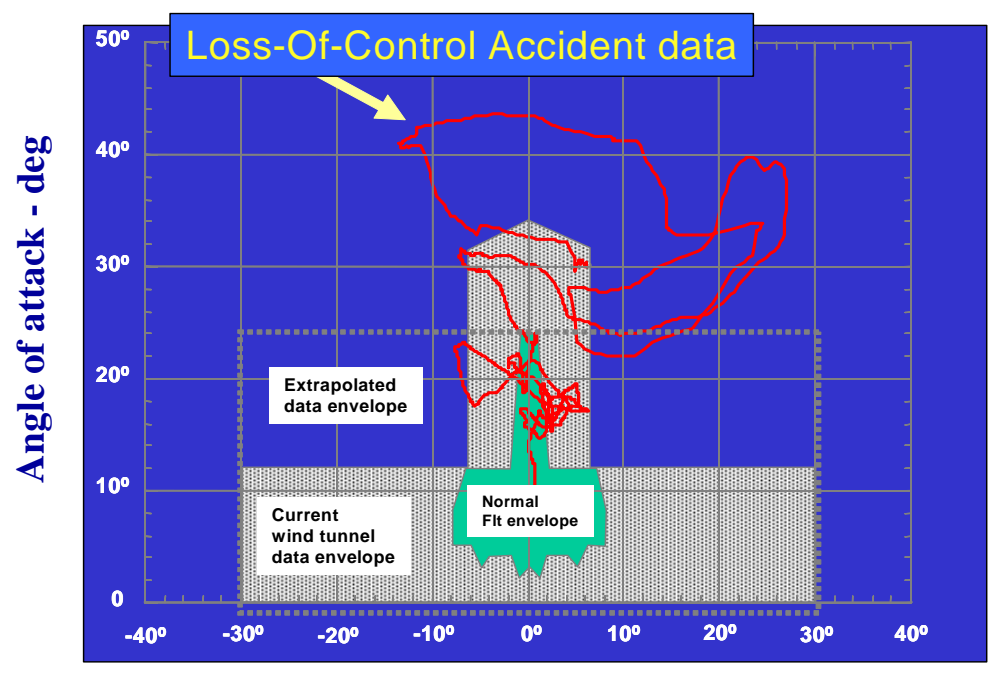

Angle of sideslip - deg

Figure 1. Transport loss-of-control accident relative to angle-ofattack and sideslip. organized as follows: Section II describes the design and research requirements for the AirSTAR testbed; Section III describes the model design, fabrication, and validation; Section IV talks about the risk mitigation effort; Section V reports the results of the first flights of a dynamically scaled transport model; and Section VI provides some concluding remarks.

\section{Design and Research Requirements}

\section{A. Scaling Requirements}

To realistically simulate the flights characteristics of a full scale aircraft, a subscale model must be scaled not only dimensionally, but also in weight, inertias, and response (including actuation and control system scaling). For the experiments that the AirSTAR testbed will be involved in, such as loss-of-control flight with high angles-ofattack and sideslip, the flow around the aircraft becomes separated and Reynolds number effects are minimized ${ }^{2}$. For more benign flight, Reynolds number effects would be significant and the aerodynamics of the model would not be representative of the full scale aircraft.

A 5.5\% model was initially considered for the GTM. This scale was chosen because of available data from previous wind tunnel tests investigating loss-of-control aerodynamics conducted at LaRC utilizing a model of this size. Building a model to this scale would enable a direct comparison and validation of the wind tunnel data with flight data. However, three questions concerning the feasibility of this testbed became apparent: 
1) "Can the dynamic scaling requirements be met with a 5.5\% model"?

2) “Can a pilot safely fly the $5.5 \%$ dynamically-scaled transport model”?

3) "Can the latency and timing requirements be met with a ground based control system"?

The first question was the focus of the initial feasibility study. Model designers first had to size the model. With a scaling factor, $K$, of 0.055 , the dimensions of the model are simply calculated as:

$$
l_{M}=K l_{F S}
$$

Where $l_{M}$ is the length of the model and $l_{F S}$ is the length of the full scale vehicle. Similar relationships are used to calculate the wingspan and other dimensions of the model. The weight of the model, $w_{M}$ is related to the scale factor raised to the third power multiplied by the weight of the full scale vehicle, $w_{F S}$ :

$$
w_{M}=K^{3} w_{F S}
$$

However, because the model will not be flying at the same altitude as the full scale vehicle, an adjustment must be made to account for the differences in the density of air at the different altitudes. This corrected model weight, $w^{\prime}{ }_{M}$ can be expressed as follows:

$$
w_{M}^{\prime}=K^{3} w_{F S} \frac{\left(d_{M}\right)}{\left(d_{F S}\right)}
$$

It can be seen from Eq (3) that model weight (assuming flight at a given altitude) is determined from full scale aircraft weight/altitude, or conversely, a given model weight can represent different combinations of aircraft weight/altitude. This is shown graphically in Fig. 2. Additional discussion of similitude and scaling requirements can be found in Ref. 4-5.

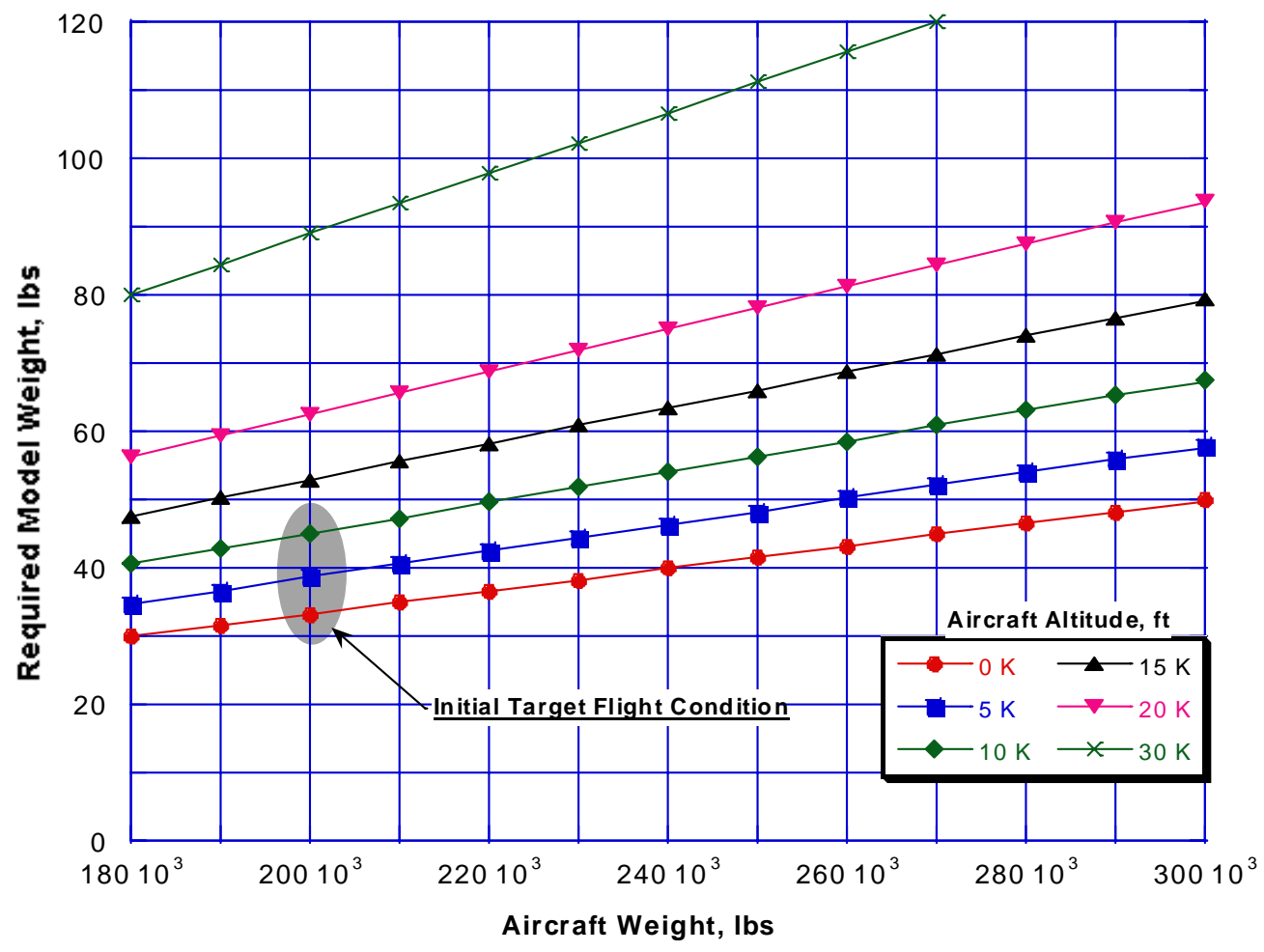

Figure 2. Model weight as a function of full-scale weight and altitude. 
Mass moments of inertia of the model are related to the inertias of the full scale aircraft by a factor of $K^{5}$. For example, the model roll inertia, $I_{\text {Mroll }}$, would be related to the full scale inertia, $I_{F S \text { roll }}$ by:

$$
I_{\text {Mroll }}=K^{5} I_{\text {FSroll }}
$$

Roll and pitch inertias are calculated similarly. As size and weight go down for a subscale vehicle, dimensions, areas, and volumes go down as expected, however the mass moments of inertia decrease greatly. This forces the design to be as light as possible and still maintain a relatively high degree of stiffness and be within $\pm 5 \%$ of the inertial targets dictated by the scaling factor for obtaining meaningful aerodynamic data. As an example, the wing should be as stiff as possible to replicate the correct aerodynamic geometry in flight yet be light enough to obtain the inertial target values set forth by the fifth order scaling factor.

The following table shows some characteristic dimensions and attributes of the model and full scale aircraft.

Table 1. Comparison of $\mathbf{5 . 5 \%}$ dynamically scaled model and full scale properties

\begin{tabular}{|l|c|c|c|c|c|c|}
\hline & Length & Wingspan & Weight & Roll inertia & Airspeed & Altitude \\
\hline $\begin{array}{l}\text { Full Scale } \\
\text { Transport }\end{array}$ & $145.5 \mathrm{ft}$ & $124 \mathrm{ft}$ & $200,000 \mathrm{lbs}$ & $2.64 \mathrm{e}^{6} \mathrm{sl}_{-} \mathrm{ft}^{2}$ & $320 \mathrm{mph}$ & $13000 \mathrm{ft}$ \\
\hline $5.5 \%$ Model & $96 \mathrm{in}$ & $82 \mathrm{in}$ & $49.6 \mathrm{lbs}$ & $1.33 \mathrm{sl}^{-\mathrm{ft}^{2}}$ & $75 \mathrm{mph}$ & $1000 \mathrm{ft}$ \\
\hline
\end{tabular}

\section{B. Control and Telemetry Requirements}

To accomplish the research objectives ${ }^{6}$, a suite of sensors, actuators, instrumentation, and controls were identified. From scaling and similitude requirements, a subscale model must respond faster than a full scale model by a factor of $\sqrt{K}$. For a 5.5\% model, this corresponds to an increase in response and control of 4.264 times that of the full scale aircraft, or just over $200 \mathrm{~Hz}$ for the fastest rates. Because of the ambitious real-time computing requirements, the limited space and weight budget of the model, and the above mentioned inherent risk involved with UAVs, it was determined that the plane would utilize a ground based control system for research maneuvers. This decision to remotely control the airplane had both benefits and drawbacks. The benefits included removing the control system from the cramped and environmentally harsh environment of the model and eliminating the risk of losing the system in the event of a crash. However, this placed additional requirements on the telemetry system to deliver data to the ground and send control signals back to the plane with minimal interruptions.

Downlink parameters required by the researchers include control surface position (measured by potentiometers on all control surfaces), DGPS position and velocities, pressure derived airspeed and altitude, heading, attitude, rates, nose camera video, and various health monitoring signals such as battery voltage. Uplink parameters include control surface and throttle commands, landing gear extend/retract commands, and DGPS corrections from the ground station. The challenge to the control system designers is to implement this strategy in response to the third question "Can the latency and timing requirements be met with a ground based control system"? Additional information concerning the development of the Mobile Operation Station (MOS) can be found in Ref. 7.

\section{Design Requirements}

With the above size and payload requirements in hand, it was now up to the model designers to determine if they could design and build a 5.5\% model which would meet the requirements. Other requirements such as structural rigidity, symmetry, flight test time, CG location, operational speeds, propulsion, video, and control surface deflection were addressed in the design. A two month feasibility study was conducted using Pro-Engineer Solid Model software to generate a solid model representation of the desired 5.5\% geometry with associated mass properties for structure and sub-systems. The results showed that the research instrumentation and a 5.5\% dynamically scaled requirement could co-exist in an aircraft of that size. This answered the first question, "Can the dynamic scaling requirements be met with a 5.5\% model”?

The design challenge is to meet the size, weight, and inertia targets set forth by the scaling factors and maintain adequate structural stiffness (i.e. minimal deflections under aerodynamic loading) and still have an adequate payload capability while meeting the required operational performance levels needed to obtain meaningful data for the customer. This design thrust starts with the creation of solid model parts and sub-assemblies using Pro-Engineer 
Solid Modeler software in an attempt to create all items, whether they be structural or commercial-off-the-shelf (COTS), needed for a complete design. All of the vehicles components are modeled as accurately as possible with regard to geometry, center of gravity, and most important weight. Sub-assemblies are then generated from these individual components to represent the wings, the fuselage, the landing gear units, the pneumatic system, etc. A final assembly can then be generated by combining all of the major components, the minor components, and the subsystem sub-assemblies. Figure 3 shows the $5.5 \%$ Generic Transport Model T1 final assembly, $25^{\text {th }}$ generation.

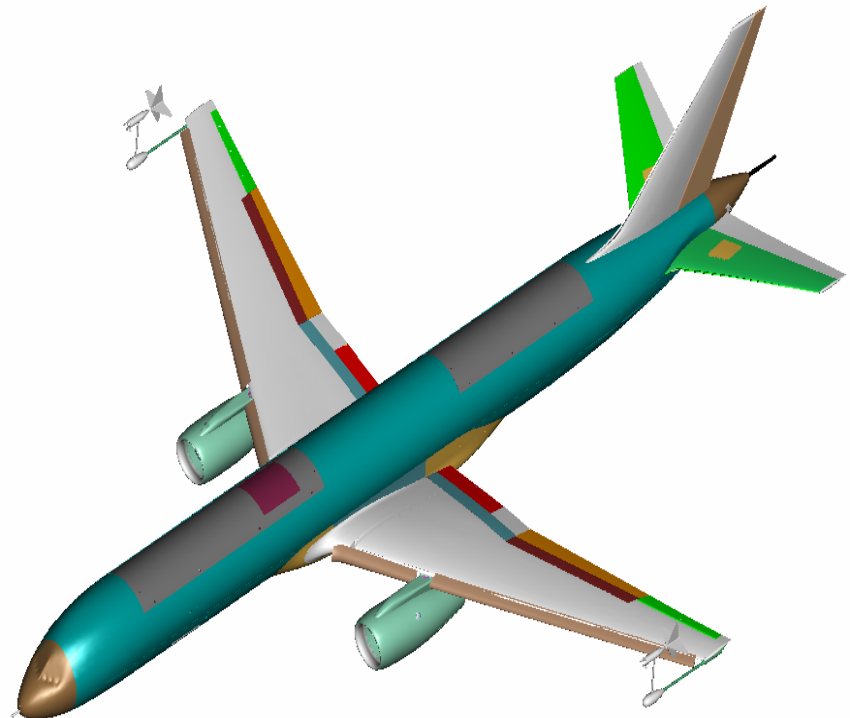

Figure 3. The 5.5\% GTM-T1 Solid Model
In Fig. 3, the model accurately represents the as-built hardware. An iterative design and fabrication effort is needed to obtain an accurate solid model for analysis purposes, hence the $25^{\text {th }}$ generation. As the individual parts are fabricated and assembled the ProEngineer Model gets updated for current weights and sizes. This level of detail includes all required fasteners, adhesives, primer, and paint (0.75 lbs.) to accurately obtain the vehicles weight and inertias. This model represents the actual hardware weight and inertia within $+/-3 \%$. The model weighs 55.85 lbs. fully loaded, ready for take-off.

The design of the GTM-T2 is similar; however with a lighter airframe, it has the capability of carrying all of the required data gathering and control electronics to enable a much greater research capacity. All weights and inertial requirements still apply for the design of the GTM-T2. Figure 4 shows the Pro-Engineer solid model of this assembly.

Notable is the fact that the vertical and horizontal tails have split control surfaces for additional flexibility in controlling the aircraft, as requested by the researchers. All control surfaces on this design utilize a custom potentiometer for control surface feedback and as a hinge point. By making sub-components work double-duty, a more efficient design can be obtained. The current fully loaded design weighs 55.16 lbs.

\section{Model Fabrication}

\section{A. Fabrication}

Model fabrication for the GTM-T1 and the GTM-T2 models was completed by the NASA LaRC Model Shop technicians under the direction of the Models Engineering Section GTM Airframe Team. The primary structure of the T1 model consists of a fiberglass and honeycomb sandwich

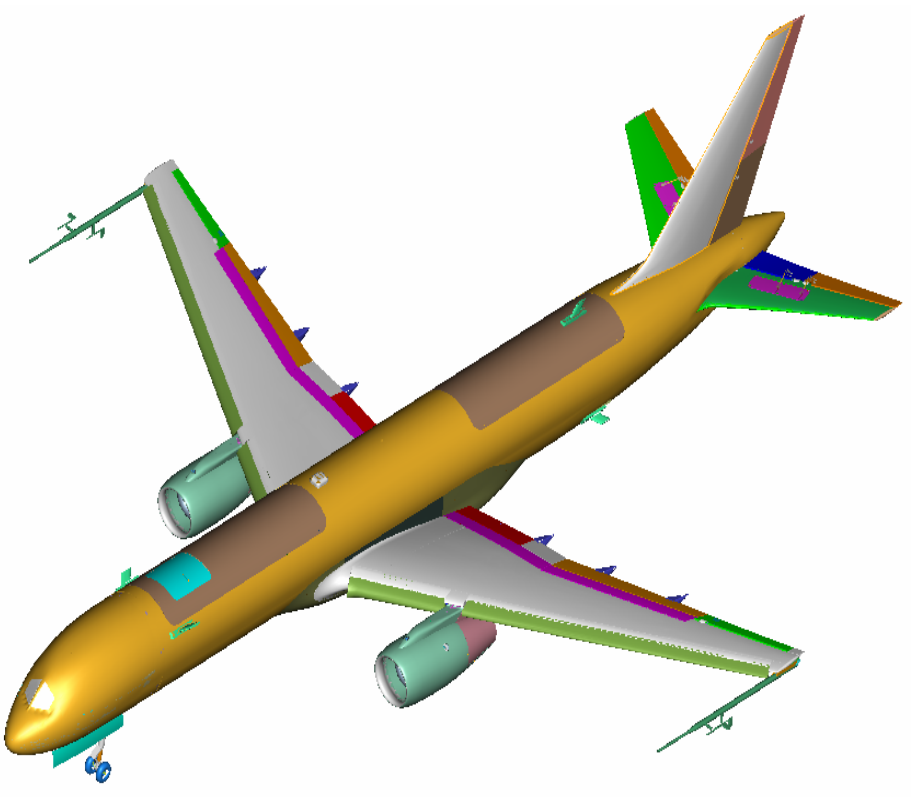

Figure 4. The 5.5\% GTM-T2 Solid Model

composite for the fuselage, along with aircraft plywood for the ribs and floor plates. The wings and empennage are fabricated of fiberglass and balsa wood sandwich composite with aircraft plywood used for spar and rib designs. 
Aluminum is used sparingly in such places as the engine pylon hard-points and the landing gear components. A picture of the "T1" during fabrication is shown below.

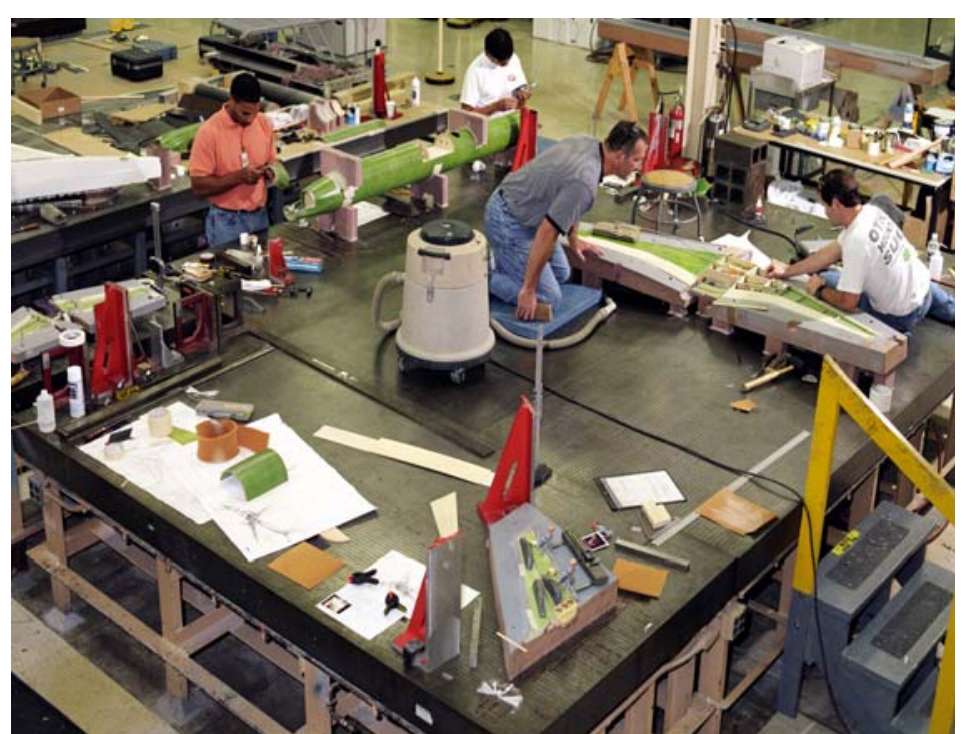

Figure 5. The 5.5\% GTM-T1 under construction
The fabrication of the GTM-T2 vehicle is similar with the exception of carbon used on the wings and empennage. The fuselage remained a fiberglass and honeycomb composite construction. Carbon was used to reduce weight, increase stiffness and strength; however, EMI characterization with the carbon components is still under investigation. Carbon was also used on the main wing spar and some sub-structure internal to the fuselage such as the nose landing gear bay. The T2 vehicle has a significantly lighter airframe which permits a heavier payload capacity for the additional research equipment required for the experiments. A top view of the completed T2 model, minus the paint and elevator servo hatches, is shown below in Figure 6.

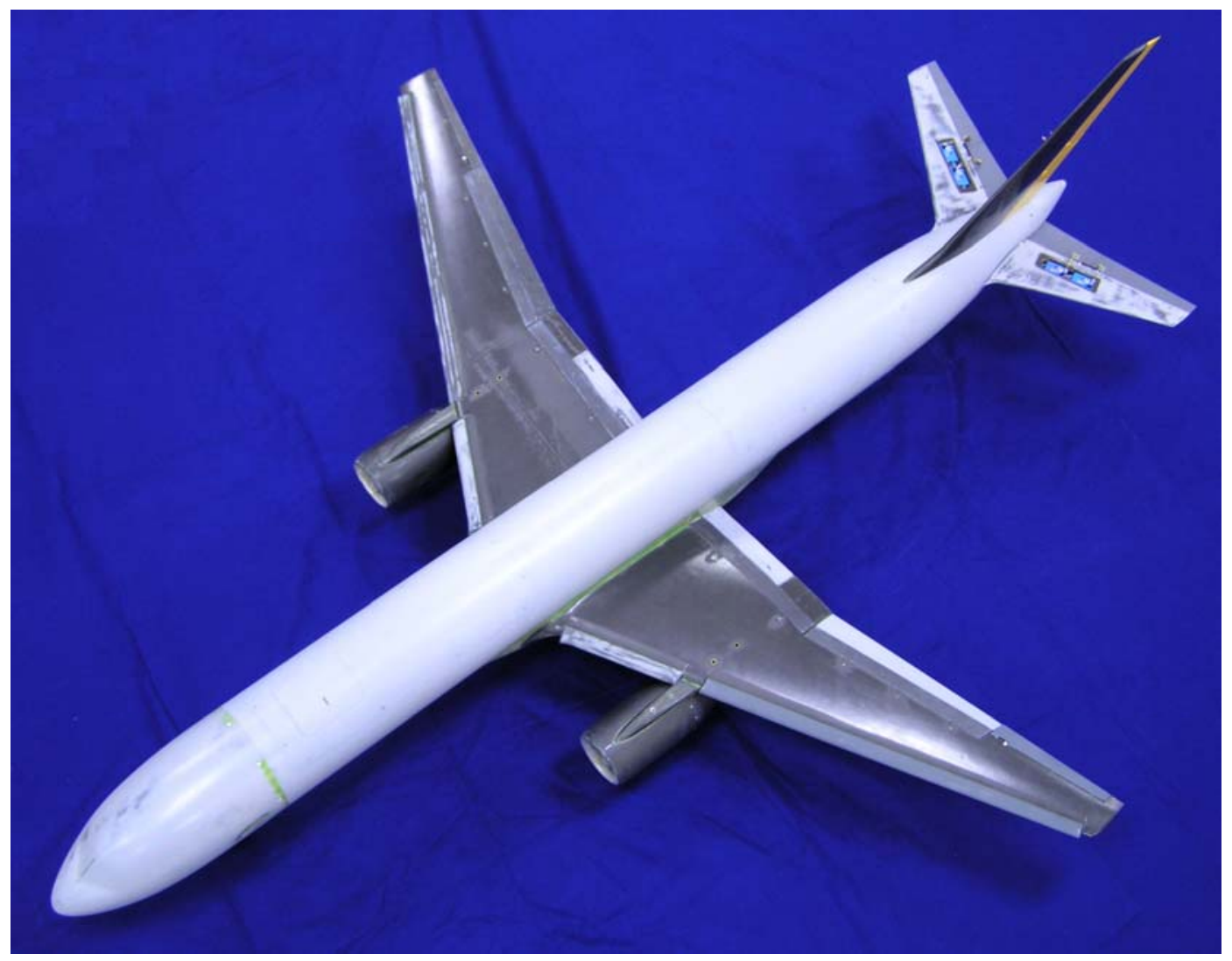

Figure 6. The completed T2 airframe. 


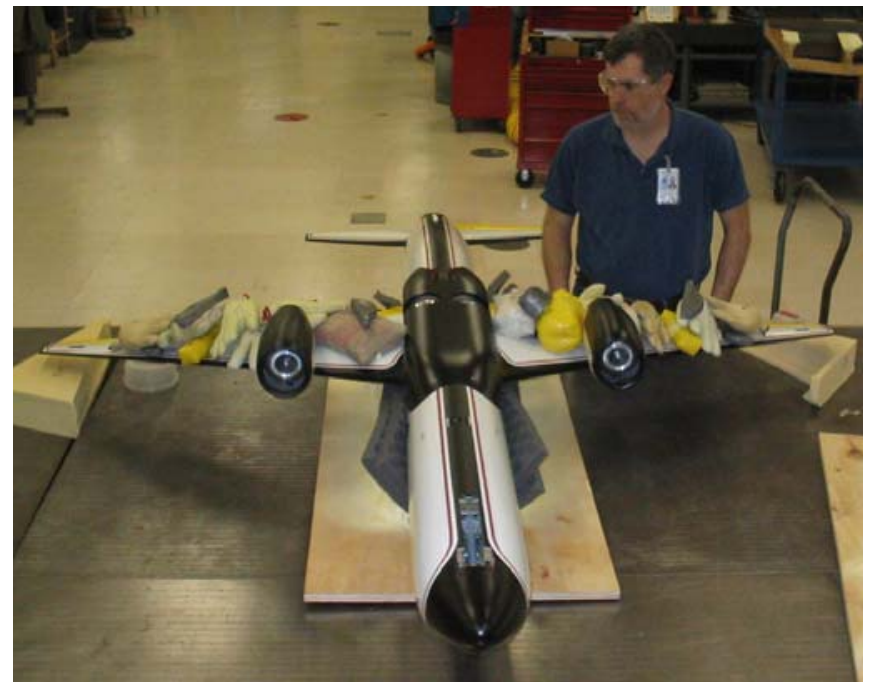

Figure 7. $5.5 \%$ GTM-T1, positive direction proof loading with a total of 195.21 lbs

\section{B. Testing}

There are three aspects of model validation testing under the GTM Project guidelines:

1. component proof load testing

2. control surfaces load testing

3. inertial testing

The GTM-T1 and the GTM-T2 models both have an associated stress analysis report. These reports also reflect the differences between the two vehicles in the areas of geometry, design, and performance. However, because of the relatively low factors of safety necessitated by the weight and performance requirements, static load testing of the airframe was required to conform to airworthiness criteria for subscale vehicles. Component proof loading was accomplished on the T1 model with the use of lead shot bags. Figure 7, shown to the left, shows the main wing undergoing positive direction proof loading.

Control surface load testing was also accomplished using dead weight. The control surface hinge moments were obtained using the equation below ${ }^{8}$ :

$$
H M=C_{s} q S \bar{c}
$$

The hinge moment coefficients where obtained from the full scale simulator software program used by the research department at NASA LaRC. Figure 8 shows the left aileron under going load testing with the wing in a positive load test direction:

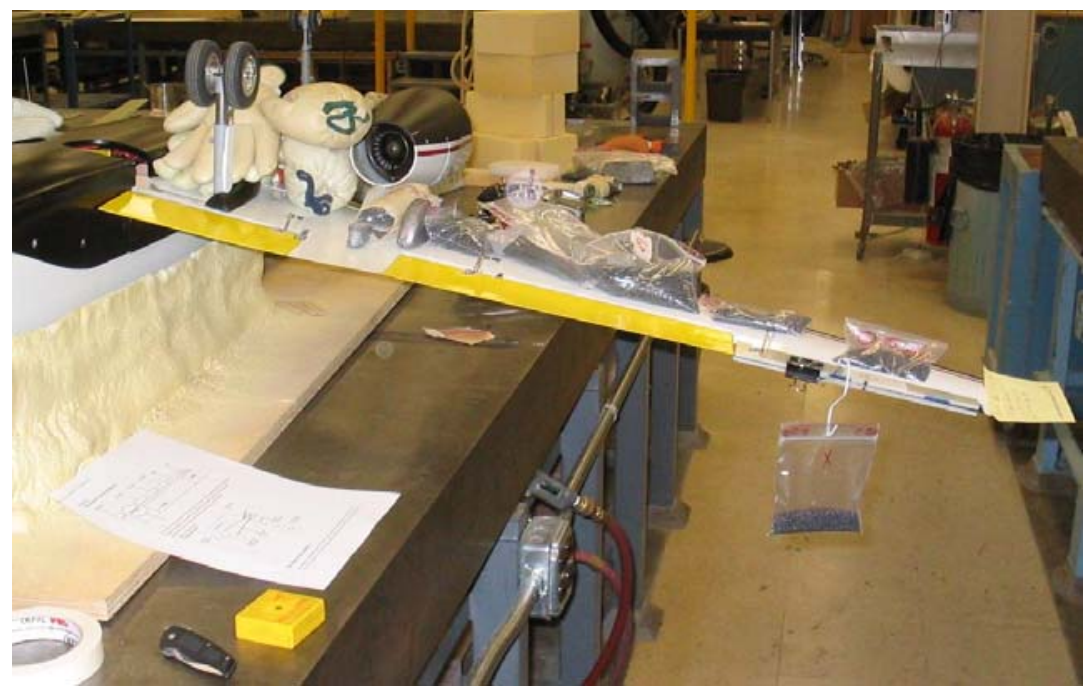

Figure 8. Left aileron load test with positive deflection.

In the above photo, the wing is loaded similar to the component proof loading effort to evaluate any hinge binding due to deflection under load, as well as to validate the actuator and its mechanical system for operational efficiency. The results of the proof and control surface loading validated the design and fabrication of the model to meet the requirements.

Inertial testing was done on the T1 model using a bifilar pendulum arrangement in three orientations: yaw, pitch, and roll. For each of these tests there was three weight points: no-fuel, target-weight, and full-fuel. The 
pendulum consists of doubled Kevlar strings suspended from an overhead I-beam in the build-up lab. The model was suspended by support straps for the yaw and pitch orientations and a $1 \frac{1}{2}$ inch diameter aluminum rod for the roll inertial tests. The roll inertial test of $\mathrm{T} 1$ is shown below in Fig. 9.

The periods of the oscillations of these tests where recorded and the inertia was calculated using the following equation ${ }^{9}$ :

$$
I=\frac{W A^{2} t^{2} g}{16 \pi^{2} L}
$$

Once the inertia was calculated using the above equation, the air damping effects where subtracted for the final inertial value. The air damping model (ADM) correction value is obtained from the model shown below in Fig 10.
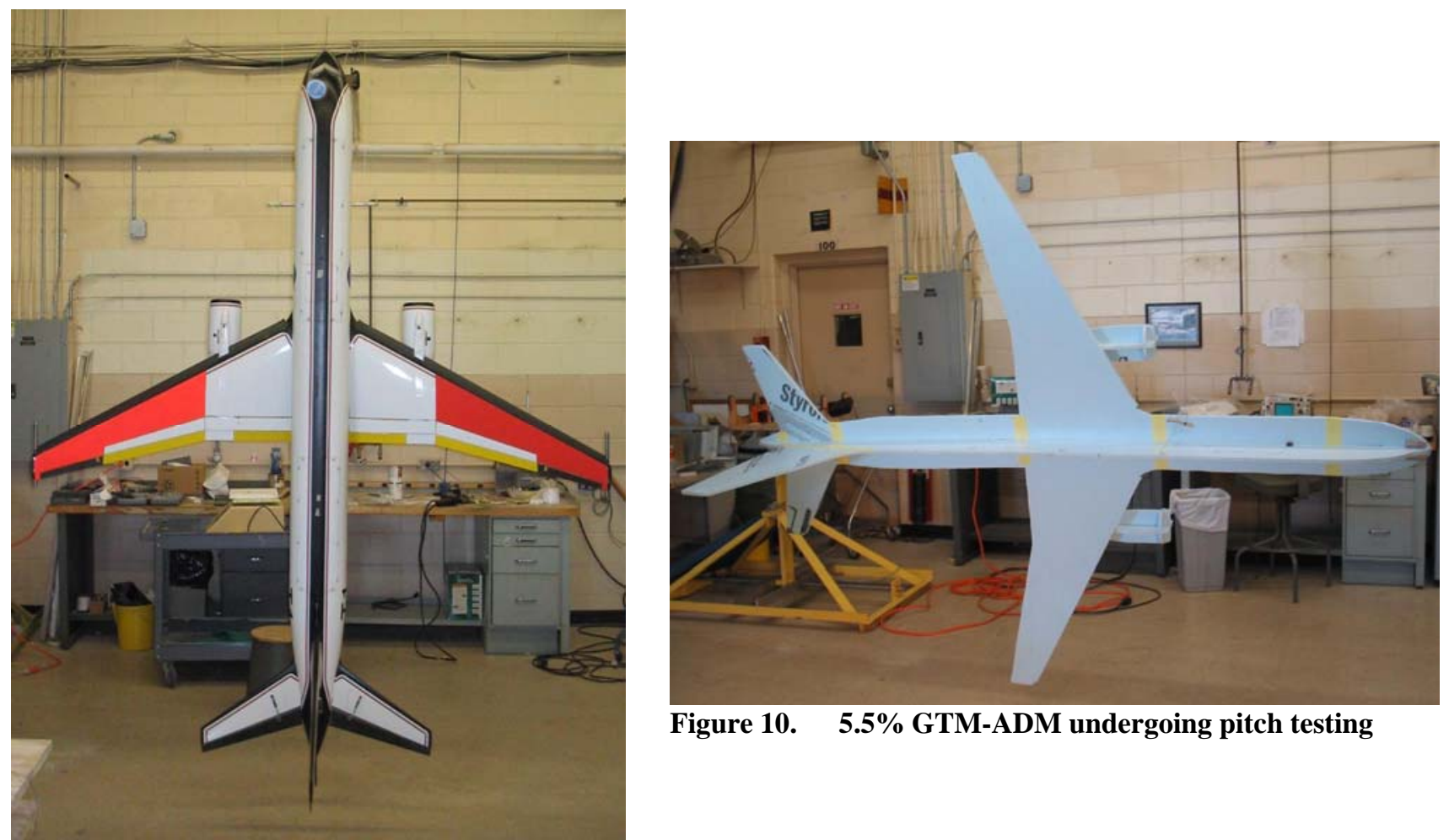

Figure 10. $\quad 5.5 \%$ GTM-ADM undergoing pitch testing

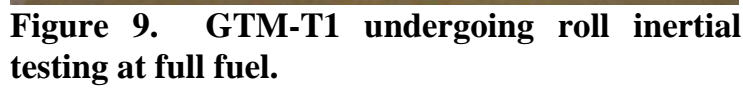

This air damping model (ADM) is a plan form representation of the $5.5 \%$ vehicle and is balanced for the target CG but weighs only 4.12 lbs. A Pro-Engineer model of the ADM was developed which would calculate the inertias of the ADM. Then a correction value was obtained by subtracting this value from the measured and calculated model value. Table 2 below compares the target weight and inertia values with those calculated from the solid model and the measured values. As can be seen from the table, the solid model estimates are very close to the measured values, which in turn provide a positive response to the first question "Can the dynamic scaling requirements be met with a $5.5 \%$ model”?

Table 2. Weight and inertia values of $\mathrm{T} 1$ aircraft.

\begin{tabular}{|c|c|c|c|c|}
\hline & Weight, lbs & Yaw inertia, lb-in ${ }^{2}$ & Pitch inertia, lb-in ${ }^{2}$ & Roll inertia, lb-in ${ }^{2}$ \\
\hline Research Targets & 49.6 & 25252 & 19696 & 6144 \\
\hline Solid Model & 49.64 & 25104 & 19756 & 6193 \\
\hline Measured & 49.64 & 25709 & 20369 & 6132 \\
\hline $\begin{array}{l}\text { Error \% (Target } \\
\text { vs. Measured) }\end{array}$ & -0.08 & -1.81 & -3.42 & 0.20 \\
\hline
\end{tabular}




\section{Risk Mitigation}

While the design requirements of a dynamically scaled transport vehicle present many engineering and fabrication challenges, operating the aircraft in a safe and efficient manner presents its own challenges. Flying and operating UAVs is an inherently risky venture, even for benign flight conditions. For this reason, the GTM team developed a risk mitigation plan that addressed the risks in the development of the hardware, operations, and pilot proficiency. This strategy is shown graphically in Fig 11.

The basis of the project's risk reduction plan is the development of a stable of remotely piloted aircraft that start with (COTS) propeller and ducted fan powered platforms (Phase 1), then advance progressively through single turbine COTS trainers (Phase 2), and finally to custom, dual turbine, swept wing platforms including dynamically scaled transports (Phase 3). The project designed and built two dynamically scaled aircraft. The first, designated $\mathrm{T} 1$, is used strictly for pilot training and carries the minimum amount of telemetry and control electronics. The second, designated $\mathrm{T} 2$, is the research aircraft. It has a lighter airframe which makes possible a heavier payload for the data gathering, telemetry, and control electronics required for the research experiments. In addition to migrating through different types of aircraft, the risk mitigation plan also addressed wing loading as a factor in training. The T1 and T2 aircrafts have a wing loading of approximately $142 \mathrm{oz} / \mathrm{ft}^{2}$. Trainer aircraft were loaded with weight to work up to this value. This course of increasingly complex, sophisticated, and expensive

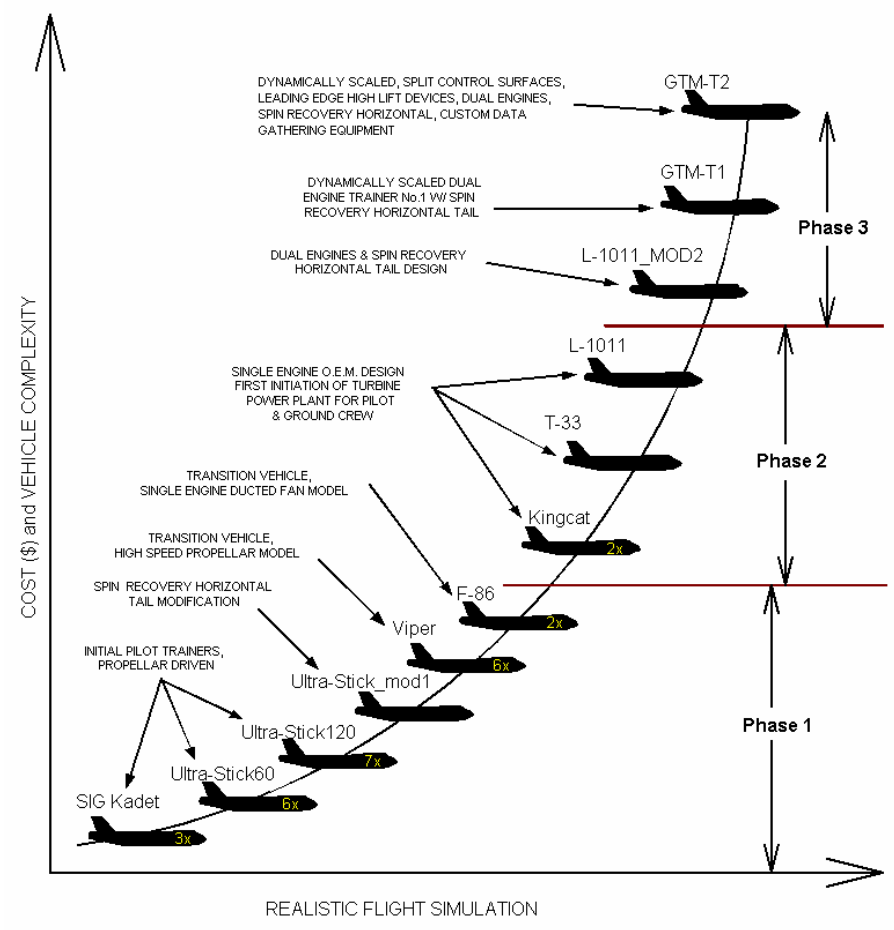

Figure 11. Airplanes used in pilot training program aircraft provided a path for the GTM team to gradually but steadily enhance it's proficiency to fly and operate subscale turbine aircraft.

Using data from previous wind tunnel tests along with as-built data from the fabrication team, a non-linear six degree-of-freedom model of the GTM was developed and incorporated into a real-time pilot simulation tool. This PC based simulator was used by the pilots to evaluate the flight handling characteristics of the dynamically scaled aircraft. It also allowed the pilots to practice flight procedures during degraded performance conditions.

A strategic decision was made early on in the project to develop the necessary pilot capability using in-house personnel. One of the major factors in this decision was the intimacy to the project that in-house pilots would bring. While outside pilots certainly would have brought an immediate capability to the project, the use of in-house personnel who also posses a strong research background brought a unique and necessary skill mix to the project. This decision produced a longer and more extensive pilot training program, but has resulted in an in-house capability that has served this project well and will certainly provide additional benefits to other RPV programs at LaRC in the future. And while there are many different areas of risk in the program, the ability and skill of the pilots, especially in take-offs and landings, was identified early on as the dominant area of risk.

While the pilots worked their way up the risk mitigation curve, the operations team used this opportunity to develop and hone their procedures, checklists, and safety guidelines. Other than a few members who had experience flying hobbyist-type RC airplanes, most of the operations and safety team had little to no previous experience with remotely piloted airplanes. And no one had any experience with the small turbines utilized by the program. All of the project procedures, checklists, and safety guidelines continually evolved during this training process to the safe and efficient form that they now have. 


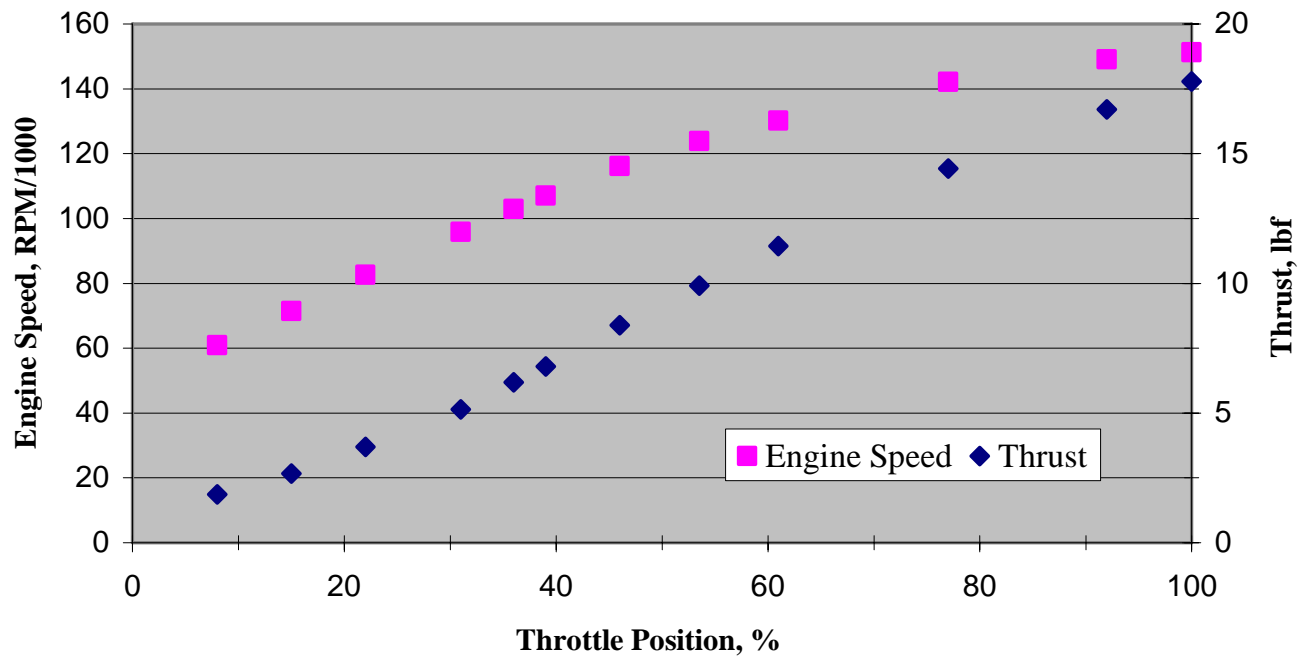

Figure 12. Engine response as a function of throttle input

This path also allowed the team to test hardware and software in the laboratory and on proven airframes before installing it in more complex aircraft. The turbine engines and servo actuators used by the project were subjected to various static and dynamic tests prior to being installed in the aircraft. Figure 12 is a typical plot of the thrust of the engine response as a function of throttle input. This testing allowed the team to not only characterize the individual components, but also to become familiar with the operation and handling of the equipment. This was especially critical with the turbines as specific procedures were developed for startup, operation, and shutdown of the engines. These initial procedures were then refined as the team accumulated more time in the field with the engines.

In addition to the many internal efforts to reduce risk, other guidance from outside the project also played a roll in risk mitigation. One area that the project utilized was the published guidelines of the Academy of Model Aeronautics (AMA) in relation to flying subscale turbine aircraft. In the absence of other direction, the team decided to follow the training guidelines of the AMA to obtain turbine waivers for each of the in-house pilots. This included accumulating flights on certain high speed aircraft and flying under the supervision of a current turbine waiver holder (using a "buddy-box" setup). After some preliminary training locally, the team spent a week training under an experienced veteran turbine pilot and received their turbine waivers. Additional input was received from reviews conducted by Langley's Airworthiness and Safety Review Board, the Wallops Flight Facility UAV review team, and the Federal Aviation Administration (from whom the project received a Certificate of Authorization).

All of the trainer aircraft utilized in the risk reduction program are flown strictly by a ground based pilot using a COTS $72 \mathrm{Mz}$ handheld transmitter, while keeping the plane within eyesight. With the T2 vehicle, the main research maneuvers will be conducted by the research pilot stationed in the MOS. However, for takeoffs, landings, and as an additional safety measure, a pilot (and co-pilot) will be on the ground with the same type of transmitters that are utilized in the pilot training program. The role of the safety pilot provides an independent control link to the aircraft. The safety pilot will also have the authority to transfer control of the aircraft, via a switch on his transmitter, between himself and the research pilot. Additionally, an image from a ground based tracking system will be available to the safety pilot should he have to take control of the aircraft when it is beyond eyesight.

The risk mitigation plan developed and followed by the project has resulted in the establishment of the capability to safely fly and operate turbine powered UAVs at LaRC. All of the pilots at Langley have earned a turbine waiver from the AMA, and in total have successfully flown over 425 turbine flights since the start of the program.

\section{Flight Testing}

After conducting several low and high speed taxi tests to characterize the ground handling characteristics of the T1 aircraft, performing numerous flights on the simulator, and flying various types of trainer RPVs, it was time for the airworthiness tests of T1. The GTM team performed the initial T1 flight tests on May 27, 2005. The results of these tests would answer the second question, "Can a pilot safely fly the 5.5\% dynamically-scaled transport model"? At the end of the day, after the second of the flight tests, the answer was clearly "Yes." 
The first flight was scripted to simply take the aircraft off, fly around in an oval pattern with the landing gear still deployed, let the pilot trim out the aircraft and get a feeling for performance, and then land the aircraft. However, soon after taking off, the aircraft exhibited a strong tendency to pitch up. After several pitch oscillations, the pilot was able to trim out enough of the pitching moment to gain positive control of the plane and land it safely. Once on the ground, the pilots and ground crew consulted and came to the conclusion that the nose up tendency of the T1 was due to the adjustable horizontal stabilizer being set with too much nose-down attitude. Before the flight, there had been some discussion as to the reference used to set the angle of the horizontal stabilizer, and it was positioned an additional $2.66^{\circ}$ leading edge down. To remedy the pitch up moment, the team agreed to adjust the stabilizer back to its original setting. This diagnosis proved to be correct, and the second flight went very smooth with the T1 flying around for several minutes before finally coming to an uneventful landing on the 6000' runway.

Three additional test flights were conducted on July 20. The intent of these follow-on flights is to allow other project pilots to fly the aircraft and transition it from an experimental platform into the pilot training program. Once the pilots have gained the confidence and skills necessary to routinely fly the T1, transitioning to the T2 research vehicle should be practically transparent. Fig. 13 shows the T1 aircraft taking off and Fig. 14 is the aircraft in flight.

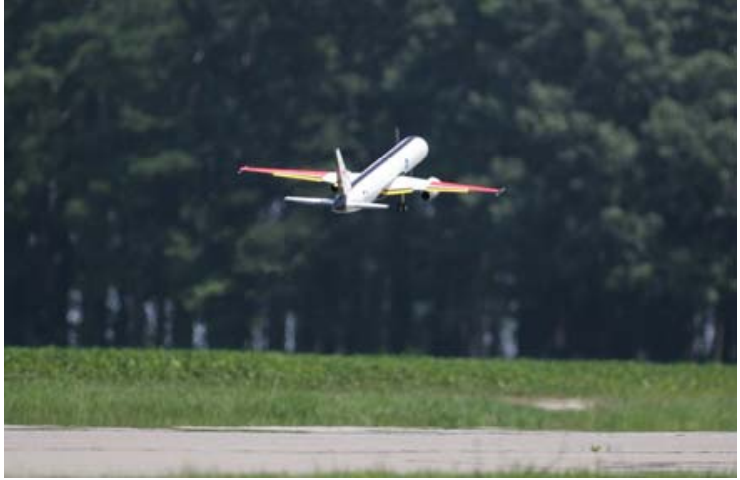

Figure 13. T1 aircraft taking off

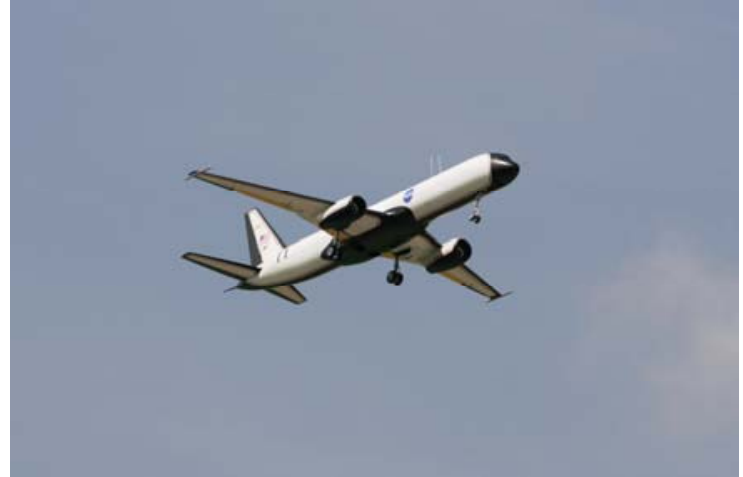

Figure 14. T1 aircraft in flight

\section{Conclusion}

Loss of control events are a major cause of US commercial airline crashes. In response to the White House Commission on Aviation Safety and Security's report to the President, NASA is developing technologies to reduce the number of these types of events. One of the tools being developed at NASA LaRC is a dynamically scaled flying testbed that will be used to validate these technologies. Because of the risk associated with putting a full scale vehicle into the type of maneuvers required for validation, a remotely piloted subscale vehicle and associated ground based control system are being developed. The testbed will be used to help build expanded flight simulators and to test new algorithms used to prevent and detect impending loss of control events. The researchers at LaRC have successfully designed and flown a dynamically scaled transport model, and are now in the process of outfitting the model and ground station for the research flights. This research, in association with other AvSSP projects sponsored by NASA, will provide a safer experience for the air traveling public.

\section{References}

\footnotetext{
${ }^{1}$ Gore, A.A, "Final Report to President Clinton - White House Commission on Aviation Safety and Security”, February 12, 1997

${ }^{2}$ Croft, J. W., "Refuse to Crash - NASA Tackles Loss of Control,” Aerospace America, March 2003

${ }^{3}$ Jordan, T. L., Langford, W. M., Belcastro, Christine M., Foster, J. M., Shah, G. H., Howland, G., Kidd, R., "Development of a Dynamically Scaled Generic Transport Model Testbed for Flight Research Experiments”, AUVSI Unmanned Unlimited, AUVSI, Arlington, VA, 2004

${ }^{4}$ Gainer, T.G. and Hoffman, S. "Summary of Transformation Equations and Equations of Motion Used in Free-Flight and Wind Tunnel Data Reduction and Analysis”, NASA SP-3070, 1972

${ }^{5}$ Wolowicz, C.H., Bowman, Jr., J.S., and Gilbert, W.P., "Similitude Requirements and Scaling Relationships as Applied to Model Testing”, NASA TP-1435, 1979

${ }^{6}$ Foster, J.V., Cunningham, K., Fremaux, C.M., Shah, G. H., Stewart, E. C., "Dynamics Modeling and Simulation of Large Transport Airplanes in Upset Conditions” (submitted for publication)
} 
${ }^{7}$ Bailey, R.M., Hostetler, R.W., Barnes, K.N., Belcastro, Christine M., and Belcastro, Celeste M., "Experimental Validation: Subscale Aircraft Ground Facilities and Integrated Test Capability” (submitted for publication)

${ }^{8}$ Stone, R. W., and Burk, S. M., "Hinge Moment Characteristics of Balanced Elevator and Rudder for a Specific Tail Configuration on a Fuselage in Spinning Attitudes” NACA TN 1400, August 1947

${ }^{9}$ Miller, M. P., “An Accurate Method of Measuring the Moments of Inertia of Airplanes” NACA TN 351, October 1930 